\title{
Persistent periodic hiccups following brain abscess: a case report
}

\author{
P H P Jansen, E M G Joosten, H M Vingerhoets
}

\begin{abstract}
A case is reported of a patient with periodic persistent hiccups and secondary generalised epilepsy lasting for a period of five years following a right temporal brain abscess. The recurring episodes of hiccups had a ten day rhythmicity and unlike epileptic convulsions were unresponsive to treatment.
\end{abstract}

Hiccuping is one of the first movements of the human embryo and starts in the eighth week of gestation. Their purpose is unclear. Hiccups may represent a vestigial element of a primitive reflex, the significance of which is now lost. Alternatively, it may represent an early respiratory movement that helps prepare the fetus for postpartem breathing. ${ }^{12}$ During fetal life and after birth the frequency of the hiccups gradually decreases. ${ }^{3}$ Exceptions to this rule are people who experience persistent hiccups, that can last for more than $\mathbf{4 8}$ hours and recur at frequent intervals. ${ }^{14}$ Some people have been known to hiccup for more than 60 years. ${ }^{5}$

We describe a patient who has had persistent postencephalitic hiccups for a period of five years. His bouts of hiccuping lasted for ten days and alternated with hiccup-free intervals of equal duration. To our knowledge this ten day biorhythmicity has not been previously reported.

\section{Case Report}

A 44 year old white male had been in good health until he was involved in a serious traffic accident at the age of 20 . He sustained multiple skull fractures, a severe contusion of the right cerebral hemisphere and lesions of the first eight cranial nerves on the right side. His recovery was complete, except for the cranial nerve damage.

At the age of 38 he suffered from pneumococcal meningo-encephalitis, possibly associated with the old basal skull fractures. A brain abscess developed in the right temporal lobe followed by a convulsive status epilepticus with focal motor seizures of the left arm and the left side of the face. These complications were treated with systemic antibiotic and anticonvulsant therapy. Phenobarbitone and phenytoin were started to suppress an ensuing secondary generalised epilepsy.
Recovery from this illness was incomplete and the patient was discharged from his job as medically unfit. His seizures were rare and mostly provoked by stress.

$\mathrm{Six}$ months later the patient was woken at night by rapid hiccups with a frequency of 30 per minute, which continued during his sleep. The bouts of hiccuping invariably lasted between 9.5 and 10.5 days and nights, alternating with $9 \cdot 5-10.5$ hiccup-free days and nights. Single precise and ryhthmic hiccup-sounds could be heard, accompanied by abrupt movements of the respiratory musculature. These were independent of respiratory cycle, respiratory rate and time of day. Fluoroscopy showed that the diaphragmatic movements were bilateral.

During the bouts of hiccups the patient had trouble falling asleep and awoke early. As a result of the hiccups he had a poor appetite leading to loss of weight. During hiccup-free intervals his appetite returned. The frequency of the hiccups decreased slightly towards the end of each episode.

Vomiting and gagging prolonged the interval between successive hiccups. The hiccups were not influenced by other methods of vagal stimulation, by irritation of the mouth and pharynx, interruption of respiratory rhythm, exercise, changes of body temperature and psychotherapeutic approach. Trials with chlorpromazine, haloperidol, methylphenidate, phenytoin, valproic acid, metoclopramide, lithium, tryptizol and amantadine all failed. As barbiturates are capable of inducing hiccups phenobarbitone medication was replaced. Phrenic block was not tried because the diaphragmatic movements during hiccups were bilateral. Biochemical and endocrinological assays of cerebrospinal fluid, blood and urine during the bouts of hiccups and hiccup-free intervals were within normal limits.

Repeated EEG examinations during the hiccup-bouts and hiccup-free intervals revealed left frontotemporal slowing as well as slight right temporal slowing decreasing with time. Nasopharyngeal electrodes failed to show any additional temporal abnormality. Cerebral MRI scanning showed extensive pathological changes in the right temporal lobe and right frontobasal areas. (fig). The brainstem and structures around the medulla were intact. The ventricles were of normal size. 
Figure MRI; slice at the level of the right temporal lobe $S E 3000 / 120, N E X$ $2,128 \times 256$; showing extensive changes of the temporal lobe with high $S I$.

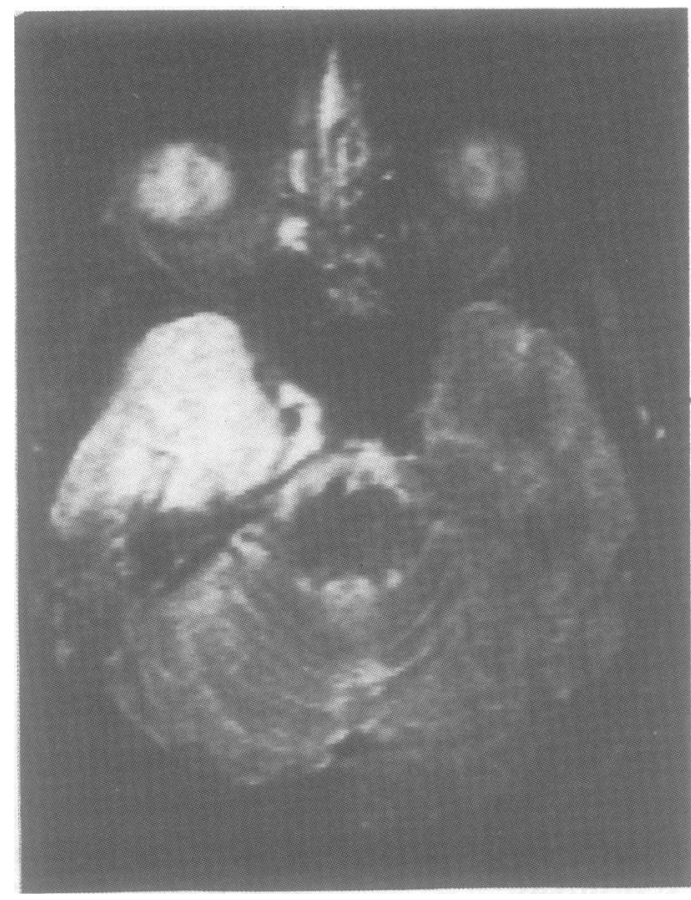

\section{Discussion}

The fact that episodes of hiccups may continue and start or stop during sleep is a strong argument in favour of an organic aetiology. The association of secondary generalised epilepsy and periodic hiccups suggest that the bouts of hiccups may represent periodic epileptic episodes. Hiccups and epilepsy may both originate from central nervous system disease. Furthermore, hiccups in patients with epilepsy may result from anticonvulsive medication.

Although it has long been recognised that epileptic seizures occur non-randomly, documented evidence concerning biorhythms and epilepsy have been surprisingly rare. It is well known that many patients show an altered seizure-liability or interictal EEG discharge rate at night, in the early morning, or during REM sleep. It is unclear how far this reflects underlying circadian or ultradian rhythms, masks the effects of sleeping and waking, or of REM and non REM-sleep cycles. ${ }^{6}$

Paroxysmal EEG discharges during bouts of hiccups may be undetectable on the scalp if it is due to a small focus in a buried cortical sulcus or in a deep limbic or subcortical regions. The failure however, of anticonvulsive medication to suppress hiccups while completely controll- ing seizures does not support an epileptic origin of hiccups. Bouts of hiccuping lasting several hours for about seven consecutive days followed by an interval of about a week in which none or very few hiccups were observed were described in a retarded epileptic male. ${ }^{7}$

To our knowledge, a ten day biorhythmicity in humans has not been previously reported. This biorhythm represents the hypothetical influence of higher neural centres on a hypothetical hiccup-reflex arc, whose central connections are located in the brainstem or upper cervical segments of the spinal cord. ${ }^{127}$ Adults are less prone to hiccups and this suggests that this is due to increased inhibition by these higher centres upon the hiccup-reflex arc Central nervous system causes of hiccups may release this inhibition. In our patients the pathological changes and atrophy of temporal and frontobasal structures following a right temporal brain abscess may therefore have released inhibitory efferents to the brainstem and upper cervical spinal cord.

This, however, does not explain the periodic loss of inhibition. Clarification of this theory would require the use of depth EEG, but this investigation must be restricted to the small number of patients who are candidates for neurosurgery.

Hiccups remains an intriguing problem presenting many unexplained clinical features, including ten day biorhythmicity.

We thank Professor J Valk for the cerebral MRI scanning and Professor B P M Schulte and Dr C D Binnie for their valuable advice.

1 Lewis JH. Hiccups: Causes and Cures. J Clin Gastroenterol 1985;7:539-52.

2 Newsom Davis J. An experimental study of hiccups. Brain 1970;93:851-72.

3 Anthoney TR, Anthoney SL, Anthoney DJ. On temporal structure of human hiccups: ethology and chronobiology. Int J Chronobiol 1978:5:477-92.

4 Soundian JV Cain JC Intractable hiccup: etiologic factors in 220 cases. Postgrad Med 1968;43:72-7.

5orld Records, 22nd ed. New York: Sterling, 1984:33.

6 Binnie CD. Biorhythmicity modulators of seizure events in generalised epilepsies. In Abstracts of the 16th International Epilepsy Congress Hamburg. Berlin, Feese and Schulz, 1985

7 Van Heuven PF, Smeets PM. Behavioural control of chronic hiccupping associated with gastrointestinal bleeding in a retarded epileptic male. J Behav Ther Exp Psychiat 1981;12:341-5. 